\title{
Special Issue on mild behavioral impairment and non-cognitive prodromes to dementia
}

This Special Issue provides a systematic examination of the neuropsychiatric symptoms (NPS) and non-cognitive prodromes of dementia, with an eye toward validating the construct of mild behavioral impairment (MBI).

NPS, also referred to as behavioral and psychological symptoms of dementia, are a common noncognitive hallmark of neurodegenerative disorders, irrespective of disease etiology (Lyketsos et al., 2011). Their presence is linked to more rapid cognitive decline, earlier institutionalization, and higher mortality rates (Lanctôt et al., 2017). Increasingly, NPS are being cited as an intrinsic aspect of dementia prodromes, and as a marker of impending cognitive decline that precedes the onset of cognitive symptoms (Mortby and Anstey, 2015). Thus, NPS may be an early, and potentially novel, target for intervention.

MBI describes the later-life onset of sustained and meaningful NPS, of any severity, in individuals who do not yet exhibit dementia or who exhibit no cognitive symptoms. MBI is a neurobehavioral syndrome that describes an at-risk state for incident cognitive decline and dementia; MBI can precede or emerge in concert with mild cognitive impairment (MCI). Its assessment is operationalized through the International Society to Advance Alzheimer's Research and Treatment - Alzheimer's Association (ISTAART-AA) research diagnostic criteria, developed by an ISTAART expert panel (Ismail et al., 2016), and the MBI checklist (MBIC, www.MBItest.org) - a rating scale developed specifically for MBI case ascertainment (Ismail et al., 2017). According to the operationalized criteria, MBI is hallmarked by changes in behavior or personality that start after the age of 50 years, representing a clear change from the person's usual behavior or personality. MBI is evidenced by one or more of the following: decreased motivation and drive, affectivelemotional dysregulation, impulse dyscontrol, social inappropriateness, and abnormal perception or thought content.

Using the operationalized diagnostic criteria, Mortby et al. (2018a) reported in this issue the first population-based prevalence estimates of MBI in 1,377 older adults (aged 72-79 years). MBI criterion A symptoms were reported in $34 \%$ of the population across the spectrum from cognitively normal to MCI. Irrespective of the level of cognitive impairment, impulse dyscontrol and decreased motivation were the most frequently reported MBI symptoms.

In a clinical sample of 282 memory clinic patients with MCI and subjective cognitive decline (SCD), Sheikh et al. (2018) reported a high frequency of MBI criterion A symptoms (82\%). These symptoms were associated with 3.5 higher levels of caregiver burden, thus demonstrating the clinical significance of MBI domains. The findings are of particular importance when considering support mechanisms, the need for family respite care, and the need to include the families in assessments in clinical and pre-clinical settings.

Cieslak et al. (2018) presented case studies that illustrate typical presentations of NPS in cognitive clinics, along with neuroimaging findings. These cases emphasize the challenge in primary care of identifying psychiatric symptomatology as part of neurodegenerative disease, as opposed to "typical" psychiatric illness with which clinicians are more familiar. This symptom misattribution often results in unsuccessful treatment of the psychiatric symptoms, with resultant delays to cognitive specialist referral during the treatment process. The cases further illustrate how the MBI-C can be used in a clinical context to help with differential diagnosis of later-life onset psychiatric symptoms, leading to early detection with assessment of dementia risk.

Several papers of this Special Issue address in detail the MBI domains. Reporting on decreased drive and motivation, Sherman et al. (2018) provided a comprehensive literature review of apathy in pre-dementia states. The authors conclude that there is a need to improve understanding of the neurobiological mechanisms of apathy in MCI and MBI to provide effective treatment to apathetic patients in prodromal dementia. Ismail et al. (2018) provided a scoping review of affective and emotional dysregulation symptoms, to explore the epidemiology and neurobiological links between affective and emotional symptoms and later cognitive decline. Findings from this paper highlight the prognostic utility of affective symptoms, and 
the great importance of understanding the natural history and age of onset of affective symptoms as they relate to neurocognitive disorders.

In their paper relating depression and anxiety to cortical amyloid deposition in cognitively normal elderly, Krell-Roesch et al. (2018) provided evidence of an association between anxious and depressive symptoms and cortical amyloid deposition. In a large sample of 1,038 cognitively normal elderly participants in the populationbased Mayo Clinic Study of Aging, 379 were amyloid positive. Anxiety was reported in $6.1 \%$ of this cognitively normal community sample, and depression in $7.3 \%$. Despite the low prevalence of self-reported anxiety and depression, the results support further need for longitudinal investigation of this association.

Addressing the domain of social inappropriateness, Desmarais et al.(2018) provided compelling evidence to highlight the importance of social inappropriateness as a first clinical sign of neurodegenerative disease, prior to noticeable cognitive impairment. The authors describe this as an underappreciated issue in pre-dementia populations, recommending more research into this often overlooked feature of MBI.

Finally, Fischer and Agueria-Ortiz (2018) critically evaluated whether abnormal perception or thought content is a risk factor, prodrome, or cause of dementia. Their paper focuses on psychotic symptoms in prodromal dementia, concluding that psychosis is more common than previously thought and has a negative impact on clinical course. Research is needed to improve early recognition and treatment. These contributions to the Special Issue provide strong evidence in support of the concept of MBI and provide a good framework to foster domain-specific research.

The final section of the Special Issue focuses more broadly on non-cognitive prodromes and clinical implications of MBI. Kiely et al. (2018) provided epidemiological data linking sensory loss (auditory/visual) to clinically relevant NPS in 1,393 older adults with and without cognitive impairment. Among individuals diagnosed with major neurocognitive disorders, any sensory loss was associated with $3+$ times greater rates of NPS compared to normal sensory functioning. However, the authors found no evidence of an association between sensory loss and number of NPS in cognitively healthy adults. These findings have particular relevance to the dementia care setting, indicating that individuals with sensory loss are at increased risk of NPS. In a study comparing the diagnostic and predictive utility of various Parkinson's disease (PD) MCI criteria, McDermott et al. (2018) also explored the manifestation of
NPS in PD. The authors found NPS to be more common in PD than in controls across all cognitive categories. The authors also suggest a neuropsychiatric prodrome to cognitive decline in PD, warranting further longitudinal studies with a rating scale designed specifically for prodromal PD patients, such as the MBI-C.

The last contribution to this Special Issue discusses the implications of the ISTAART-AA MBI criteria, and the MBI-C, for dementia clinical trials (Mortby et al., 2018b). With the low success rate of dementia clinical trials, which have often been limited by poor recruitment and retention of early phase illness, the argument is made that patients with later life emergence of NPS/MBI represent a potentially enriched sample of prodromal dementia. The commentary explores the utility of inexpensive large scale screening for sustained later life emergent NPS as a way of detecting this cohort for biomarker screening, and/or clinical trial enrollment. This discussion highlights the importance of NPS as part of neurodegenerative disease, in advance of cognitive impairment, as opposed to a dementia or MCIspecific phenomenon, which is still often the perspective of clinicians and researchers.

This Special Issue provides a comprehensive overview of the current research agenda to validate the construct of MBI. Studies providing a better understanding of the role and relationship of MBI with pathophysiology are needed to improve early identification, develop interventions, facilitate clinical treatment, and reduce dementia risk. Questions requiring further exploration include the prognostic utility of specific MBI domains, the role of symptom severity, and the description of NPS as a consequence of neurodegenerative disease. This exciting research agenda will increase global awareness of MBI as a syndrome, which serves as a neuropsychiatric parallel to the MCI syndrome, and may be integral to a comprehensive approach to earlier dementia detection and intervention.

\section{Moyra Elizabeth Mortby, ${ }^{1}$ Constantine G. Lyketsos, ${ }^{2}$ Yonas E. Geda ${ }^{3}$ AND ZAHINOOR ISMAIL ${ }^{4}$}

${ }^{1}$ Centre for Research on Ageing, Health and Wellbeing, Research School of Population Health, The Australian

National University, Canberra, Australia

${ }^{2}$ Department of Psychiatry and Behavioral Sciences, Johns Hopkins School of Medicine, Baltimore, Maryland, USA

${ }^{3}$ Departments of Health Sciences Research, Neurology, Psychiatry and Psychology, Mayo Clinic, Scottsdale, Arizona, USA 
${ }^{4}$ Departments of Psychiatry, Neurology, and

Epidemiology, Hotchkiss Brain Institute, University of Calgary, Calgary, Alberta, Canada

Email: zahinoor@gmail.com

\section{References}

Cieslak, A., Smith, E. E., Lysack, J. and Ismail, Z. (2018). Case series of mild behavioral impairment: toward an understanding of the early stages of neurodegenerative diseases affecting behavior and cognition. International Psychogeriatrics, 30, 273-280.

Desmarais, P., Lanctot, K. L., Masellis, M., Black, S. E. and Herrmann, N. (2018). Social inappropriateness in neurodegenerative disorders. International Psychogeriatrics, 30, 197-207.

Fischer, C. E. and Aguera-Ortiz, L. (2018). Psychosis and dementia: risk factor, prodrome, or cause? International Psychogeriatrics, 30, 209-219.

Ismail, Z. et al. (2016). Neuropsychiatric symptoms as early manifestations of emergent dementia: provisional diagnostic criteria for mild behavioral impairment. Alzheimer's E Dementia, 12, 195-202.

Ismail, Z. et al. (2017). The mild behavioral impairment checklist (MBI-C): a rating scale for neuropsychiatric symptoms in pre-dementia populations. Fournal of Alzheimer's Disease, 56, 929-938.

Ismail, Z. et al. (2018). Affective and emotional dysregulation as pre-dementia risk markers: exploring the mild behavioral impairment symptoms of depression, anxiety, irritability, and euphoria. International Psychogeriatrics, 30, 185-196.

Kiely, K. M., Mortby, M. E. and Anstey, K. J. (2018). Differential associations between sensory loss and neuropsychiatric symptoms in adults with and without a neurocognitive disorder. International Psychogeriatrics, 30, 261-272.

Krell-Roesch, J. et al. (2018). Depressive and anxiety symptoms and cortical amyloid deposition among cognitively normal elderly persons: the Mayo Clinic Study of Aging. International Psychogeriatrics, 30, 245-251.

Lanctôt, K. L. et al. (2017). Neuropsychiatric signs and symptoms of Alzheimer's disease: new treatment paradigms. Alzheimer's \& Dementia: Translational Research E Clinical Interventions, 3, 440-449.

Lyketsos, C. G. et al. (2011). Neuropsychiatric symptoms in Alzheimer's disease. Alzheimer's \& Dementia, 7, 532539.

McDermott, K. L., Fisher, N., Bradford, S. and Camicioli, R. (2018). Parkinson's disease mild cognitive impairment classifications and neurobehavioral symptoms. International Psychogeriatrics, 30, 253-260.

Mortby, M. E. and Anstey, K. J. (2015). Mental health and aging. In A. N. Pachana (ed.), Encyclopedia of Geropsychology (pp. 1-6). Singapore: Springer Singapore.

Mortby, M. E. et al. (2018a). Dementia clinical trial implications of mild behavioral impairment. International Psychogeriatrics, 30, 171-175.

Mortby, M. E., Ismail, Z. and Anstey, K. J. (2018b). Prevalence estimates of mild behavioral impairment in a population-based sample of pre-dementia states and cognitively healthy older adults. International Psychogeriatrics, 30, 221-232.

Sheikh, F. et al. (2018). Prevalence of mild behavioral impairment in mild cognitive impairment and subjective cognitive decline, and its association with caregiver burden. International Psychogeriatrics, 30, 233-244.

Sherman, C., Liu, C. S., Herrmann, N. and Lanctot, K. L. (2018). Prevalence, neurobiology, and treatments for apathy in prodromal dementia. International Psychogeriatrics, 30, 177-184. 